REVIEW

\title{
Rabies: a review of UK management
}

\author{
N McKay, L Wallis
}

Emerg Med J 2005;22:316-321. doi: 10.1136/emj.2003.013805

Rabies is endemic worldwide, and causes approximately 30000 deaths per year. In the past 20 years, 12 deaths have occurred in the UK, although all but one case were contracted overseas. We have reviewed the current literature regarding the management of possible rabies exposure in the setting of a UK emergency department. The article offers an overview of rabies, including pathology, risk assessment, and current treatment, including both preexposure and post-exposure prophylaxis. We have also included a form online, which allows the correct information to be obtained and recorded prior to seeking advice from the local virology services.

See end of article for authors' affiliations

.....................

Correspondence to: Dr N McKay, Accident and Emergency, Royal Gwent Hospital, Newport, UK docneilmckay@aol.com

Accepted 22 August 2004
$\mathrm{R}$ abies is endemic is many parts of the world. In 1998, approximately 30000 deaths in India were attributed to rabies; ${ }^{1}$ between $30 \%$ and $50 \%$ of these deaths occurred in children. ${ }^{2}$ In the UK, rabies was officially eradicated in 1920; ${ }^{3}$ however, in the past 20 years, 12 people have died from rabies in the UK. All but one of these cases contracted the condition overseas. The most recent UK death occurred in 2002, in an unvaccinated bat conservationist in Dundee who contracted a rabies virus (European bat lyssavirus (EBLV) type 2) and did not receive post-exposure prophylaxis. This case is only the second confirmed case of an EBLV type 2 infection in a human after exposure to bats. ${ }^{4}$

In an emergency department (ED), rabies is most frequently encountered in a traveller returning from abroad who has suffered an animal bite and who is seeking advice regarding risk. In 1997, 472 such individuals were referred to the Central Public Health Laboratory and received post-exposure vaccine, with or without specific immunoglobulin, mainly following dog bites in rabies endemic countries. ${ }^{5}$ Rarely, if ever, has a dog bite that occurred in the UK been felt to present a risk of rabies.

\section{PATHOLOGY \\ Virology}

Rabies is caused by a neurotropic virus of the genus Lyssavirus. Derived from the Greek for frenzy, this genus includes the classic rabies virus, two European bat lyssaviruses, an Australian bat lyssavirus, and the African Duvenhage virus. They all produce a similar fatal encephalomyelitis in humans, known as rabies.

\section{Transmission}

Because some infected animals also die from the infection, rabies is not a true zoonosis. ${ }^{6}$ A survey of rabid dogs in the USA showed that all died within 8 days (median 3) of becoming ill. ${ }^{7}$ Animals will often behave strangely once infected, demonstrating increased aggression, ataxia, lethargy, or excess salivation; nocturnal animals may become active during the daytime.

Rabies is an infection initially of wild and then domestic animals, which is spread to humans by bites, contact with mucosal membranes, and (to a much lesser extent) aerosol inhalation in bat caves. Most infections (90\%) are transmitted via domestic animals (cats and dogs), mainly due to their closer association with humans. ${ }^{8}$ There have been only three documented cases of transmission of rabies to humans from bats in Europe in the last 25 years. After the Dundee infection, 2000 bats from the UK were analysed, and only two were found to be EBL positive (both Daubeton bats). However, in mainland Europe, between 1977 and 2000, over 600 bats were found to be positive. ${ }^{3}$ The European bat lyssavirus has only been reported to be the cause of death in four patients across Europe since 1977. ${ }^{9}$

Almost all transmissions are via bites. As the virus is excreted in saliva, infection can occasionally occur via scratches infected with saliva, though the infection rate is 50 times lower. ${ }^{10}$ Human to human transmission has not been recorded, with the exception of six iatrogenic cases resulting from corneal graft implants. ${ }^{11}$ These had been taken from patients with nonspecific neurological disease, and resulted in rabies infection developing in the recipient 2239 days later, with all but one case being fatal. Increased awareness of this risk has meant that there have been no documented iatrogenic cases for the last 15 years.

Airborne transmission is thought to have occurred in two men who inhaled virus aerosols generated in caves inhabited by rabid bats, and in a laboratory worker who became infected while rabid sheep brains were being ground for vaccine production. ${ }^{12}$

The virus may be shed in breast milk, and there has been at least one suspected case of transmission from a mother to a breastfed infant. ${ }^{13}$ Transplacental infection occurs in animals but has not been reported in humans. A number of women with rabies encephalitis are known to have delivered healthy babies.

Abbreviations: CDSC, Communicable Disease Surveillance Centre; EBLV, European bat lyssavirus; ED, emergency department; HRIG, human rabies immunoglobulin 


\section{Pathophysiology}

After a bite, the virus replicates in muscle cells close to the site of the bite and then ascends to the central nervous system via the peripheral nerves. On reaching the central nervous system, there is massive viral replication on membranes within neurones. It is then transmitted directly across synapses into efferent nerves, and is deposited in almost every body tissue, including the autonomic nervous system via neural networks. It is at this stage that productive viral replication occurs with budding, particularly in the salivary glands, in preparation for the infection of other mammals.

The incubation period from bite to disease varies widely, but is usually between 30 and 90 days. Antigenic analysis has confirmed incubation periods of up to 7 years, ${ }^{14}$ although this is exceptional. Bites on the head and neck have a shorter incubation period (sometimes even as short as 15 days) compared with those on the trunk and lower extremities, due to the decreased length and greater number of neurones.

\section{Pathology}

Following infection, the brain, spinal cord, and peripheral nerves show ganglion cell degeneration, perineural and perivascular mononuclear cell infiltration, and neuronophagia. Inflammation is most marked in the midbrain and medulla in furious rabies and in the spinal cord in paralytic rabies. Vascular lesions such as thrombosis and haemorrhage are also described, mainly in the brainstem, hypothalamus, and limbic system. Outside the nervous system, there is focal degeneration of the salivary and lachrymal glands, pancreas, and adrenal medullae.

\section{SYMPTOMS AND SIGNS}

The viral prodrome is non-specific and, particularly in areas where the virus is rare, the diagnosis is often made late. In many patients, the first symptom is itching, pain, or paraesthesia at the site of a healed bite wound. Prodromal symptoms then develop, including fevers, myalgia, headache, irritability, depression, and upper airway or gastrointestinal symptoms (box 1).

A radiculopathy or other symptoms involving the bitten limb are often complained of, before encephalopathic syndrome, (of which two distinct types are described below) develops.

\section{Furious rabies}

This is the more common presentation. It manifests as irritability, agitation, and hyperaesthesia. Reported abnormalities include cranial nerve lesions, upper motor neurone lesions, and autonomic disturbances (disorders of blood pressure, hypersalivation, and sweating). The pathognomonic symptom of hydrophobia is a triad of inspiratory muscle spasm, painful laryngospasm, and terror (fear of swallowing). It initially occurs when trying to drink water but

\section{BOX 1 PRODROMAL SYMPTOMS OF RABIES}

- Itching, pain, or parasthesia at site of bite wound

- Fevers

- Myalgia

- Headache

- Irritability or depression

- Gastrointestinal upset

- Radiculopathy in bitten limb

- Proceeding to encephalopathy eventually can occur with the slightest stimulus or even at the mention of water. In its most severe form, the reflex can be provoked simply by drafts, at which point it is known as aerophobia. This reflex is combined with extension of the back and arms and may even end in a generalised convulsion, or cardiorespiratory arrest. Without supportive treatment, approximately one third of patients will die within the first few days of a hydrophobic spasm. The rest will proceed to a generalised flaccid paralysis and rarely survive more than a week without intensive care support. Even with such support, the disease is fatal within months, with very few reported cases of survival. In one report, all survivors had been given pre-exposure vaccination and post-exposure prophylaxis. ${ }^{15}$ Limited recovery is often a more appropriate term than survival, as survivors will have long term neurological deficits.

\section{Paralytic rabies}

Accounting for under $20 \%$ of human cases, paralytic rabies tends to follow either bites from vampire bats ${ }^{16}$ or bites in people who have received pre-exposure vaccination. After the usual prodrome, a flaccid paralysis develops, usually in the bitten limb, which ascends (either symmetrically or asymmetrically) with pain and fasciculation in the affected muscles. There is often mild sensory disturbance. Paraplegia and sphincter disturbances follow, until eventually a fatal paralysis of the respiratory and deglutive muscles occur. Hydrophobia is rare but may be noted as a few spasms of the laryngeal muscles in the terminal phase. These patients may survive for up to 30 days without ITU support.

\section{DIAGNOSIS}

\section{Differential diagnoses}

Rabies should be suspected in any case where a patient presents with neurological signs having been bitten by a mammal, particularly in an endemic area. Possible differential diagnoses vary, depending on whether the presentation is as furious or paralytic rabies.

\section{Furious rabies}

Differential diagnoses would include delerium tremens, botulism, diphtheria, drug ingestion (phenothiazines and amphetamines), and plant ingestion (Datura fastuosa). However, rabies should be suspected in any patient presenting with severe neurological signs having been bitten by a mammal in a rabies endemic area.

Tetanus can also follow an animal bite; wounds to the head and neck can cause cephalic tetanus. This affects the muscles innervated by the cranial nerves, causing trismus, facial stiffness, and pharyngeal and laryngeal spasms. These can cause death by asphyxia or aspiration. However, it can be distinguished from rabies by its shorter incubation time $(<15$ days), the persistence of muscle spasms between attacks, and the absence of meningoencephalitis (CSF is normal in tetanus).

BOX 2 DIFFERENTIAL DIAGNOSIS OF FURIOUS RABIES

- Delerium tremens

- Botulism

- Diphtheria

- Drug ingestion (phenothiazines and amphetamines)

- Plant ingestion (Datura fastuosa)

- Tetanus 


\section{Paralytic rabies}

This has a wider differential diagnosis owing to the slower nature of its presentation and more diffuse neurological features. Often the history is of utmost importance, as paralytic rabies can present very similarly to other causes of ascending paralysis.

Examination of CSF will distinguish between paralytic rabies and Guillain-Barré syndrome (table 1). Polio rarely has any objective sensory disturbances. Another rare form of encephalitis, herpes simiae, is transmitted by monkey bites. It has a short incubation period ( 3 days) and vesicles form around the site of the bite. ${ }^{17}$ There are also other encephalitidies secondary to arboviruses, which can produce a similar picture. However, the history of an animal bite (when available) points towards the correct diagnosis.

\section{Laboratory diagnosis}

There are two main avenues available in making the diagnosis. Firstly, the offending animal should be captured if possible. Brain biopsy provides samples of brain tissue, which can then be examined, and rabies antigen can be detected within a few hours by direct immunofluorescence or PCR. If the animal is not overtly "rabid", it can be observed for a period of 10 days. At any sign of infection or deterioration, the animal should be killed and the brain tested. Animals behaving strangely should be killed and examined as soon as possible after the bite or exposure.

In a patient presenting with suspected rabies, demonstration of viral RNA by PCR or viral antigen in skin biopsies allows the earliest diagnosis (immunofluorescence highlights it in nerve twiglets). An alternative source for investigation is corneal scrapings; however, this site is not as sensitive as a neck skin biopsy. Rabies antibodies are not usually detectable in CSF or serum until the eighth day of illness in unvaccinated patients, ${ }^{18}$ and in most cases of rabies, patients have died before an antibody response is detectable. A peripheral neutrophil leucocytosis is common in the early stages of the disease, but is not particularly helpful in reaching the diagnosis.

A recent series in France compared the sensitivies of the various tests in a premorbid situation (table 2). They all showed a high specificity approaching $100 \% .{ }^{19}$ As is evident from these values, none of the tests have a high sensitivity and thus a high degree of clinical suspicion is needed, rather than relying on a negative result.

The laboratory diagnosis of rabies pre-mortem has always been difficult, but PCR has improved the sensitivity of the tests on skin snips and saliva. Another new technique involves the inoculation of saliva into mice, which can then be examined with PCR to confirm the diagnosis.

\section{RISK ASSESSMENT}

Risk assessment is the most important initial step in management of these cases. This must include a full history, paying attention to the exact circumstances of the bite, the biting animal's behaviour, whether the bite was provoked or unprovoked, whether from a wild or pet animal, the site of the bite, and initial treatment of the wound. Of utmost

\section{BOX 3 DIFFERENTIAL DIAGNOSIS OF PARALYTIC} RABIES

- Guillain-Barré syndrome

- Polio

- Herpes simiae encephalitis

- Arbovirus encephalitis
Table 1 CSF characteristics of differential diagnoses

\begin{tabular}{lll}
\hline Diagnosis & Protein & Cells \\
\hline $\begin{array}{l}\text { Rabies and } \\
\text { Meningoencephalidities }\end{array}$ & Raised & Pleocytosis \\
Tetanus & Normal & Normal \\
$\begin{array}{lll}\text { Guillain-Barré syndrome } \\
\text { Polio }\end{array}$ & $\begin{array}{l}\text { Raised } \\
\text { Raised }\end{array}$ & $\begin{array}{l}\text { Normal } \\
\text { mild pleocytosis }\end{array}$ \\
\hline & & mil or \\
& & \\
\hline
\end{tabular}

Table 2 Sensitivity of tests for rabies

\begin{tabular}{ll}
\hline Test & Sensitivity \\
\hline Serum ELISA & 0.17 \\
CSF RT-PCR & 0.09 \\
Saliva RT-PCR & 0.30 \\
Skin biopsy immunofluorescence & 0.86 \\
\hline
\end{tabular}

importance are details of previous vaccination of the patient and animal (if bitten by a pet). The country in which the bite occurred can be compared with published risk charts, which are regularly updated by World Health Organization offices (see online Appendix). A bite from a mammal in an endemic area has to be assumed to be at risk of carrying rabies infection. Animals behaving strangely or inappropriately are a higher risk. Carnivores are seen to offer a higher risk of infection than herbivorous or omnivorous animals.

The type of exposure is also important. Bites pose a high risk, but scratches and non-bite exposure of either an open wound or mucous membrane must also be assumed to be at risk. Contact with the animal's coat or faeces is not a risk for infection.

The position of the wound is of importance when considering the urgency of post-exposure prophylaxis, and the site of the bite is important when predicting the severity of exposure. Bites to the face and neck should be treated as more serious, and merit a lower threshold for treatment, owing to the decreased length of peripheral nerves to be ascended before the virus enters the central nervous system.

Patients with "unsure exposure" should be considered to be high risk. This situation may be due to inability to provide an accurate history (the very young, elderly, or those with language difficulties), or lack of definite exposure (for example, a sleeping patient who awakes to find a bat in the room while camping in an American forest). In these cases, a high index of suspicion needs to be maintained, and patients should be treated accordingly (especially if the animal is not available for examination).

\section{PREVENTION AND CONTROL}

The main aim of rabies control is to prevent infection. In the $\mathrm{UK}$, beyond rare infection in the native bat population, there is no indigenous rabies. This is maintained by active immunisation of animals entering the UK and by strict quarantine regulations; animals from outside the UK spend 6 months in quarantine prior to being allowing into the country. However, the Pet Travel scheme introduced in February 2000 allows the passage of cats and dogs, with strict passport criteria, to enter freely from Europe and North America. These animals are identifiable, are known to be vaccinated, and have had a documented appropriate response to the vaccine. ${ }^{20}$ 


\section{TREATMENT}

The aim with suspect animal bites sustained outside the UK is to prevent development of rabies. The most important steps are thorough cleaning of the wound and use of post-exposure prophylaxis, which together reduce the risk from a known rabid animal bite from $37-60 \%$ to near zero. ${ }^{21}$

Once rabies symptoms have developed, treatment itself is mainly supportive, as the outlook is dismal. Patients must be heavily sedated in order to control their pain and terror. The mainstay of treatment is intensive care support, including paralysis, sedation, and ventilation. Ketamine has been suggested as an appropriate agent for this purpose. ${ }^{15}$ Antiserum, antiviral agents, interferon, corticosteroids, and other immunosuppressants have all proved useless.

Four patients have been documented as surviving rabies infection. ${ }^{22}$ All had some degree of pre-exposure or postexposure prophylaxis and lengthy stays in the intensive therapy unit to control other complications, including electrolyte disturbances, cardiac failure, raised intracranial pressure, convulsions, and hyperpyrexia. They have usually been left with neurological deficits. ${ }^{15}$

There are several steps in the prophylaxis of rabies, as follows.

\section{Pre-exposure vaccination}

Pre-exposure vaccination carries some degree of protection for the individual. It does not aim to provide full immunity, but does provide extra protection to allow the bitten individual time to seek administration of immunoglobulin and/or further vaccination. Pre-exposure vaccination is currently offered to those at risk of infection, either occupationally or through travel.

Currently, the course involves three doses of tissue culture vaccine given into the deltoid on days 0,3 , and 28 . The seroconversion rate is $98.2 \% .{ }^{23}{ }^{24}$ Antibody levels need not be checked unless the patient is at particularly high risk of infection (for example, rabies laboratory staff). Otherwise, boosters can be given between 6 and 24 months to prolong protection. ${ }^{24}$ Currently the recommendation for vaccination includes those at risk occupationally and those intending to travel to rabies endemic areas for periods of more than 30 days. ${ }^{25}$

By far the best means of prevention is to avoid coming into contact with rabid animals, and education for travellers to how to avoid a dangerous bite is central to prevention. Precautions include wearing long trousers, ignoring free roaming cats and dogs, and the immediate washing of wounds (see below).

Telephone advice for health professionals on pre-exposure rabies vaccination can be obtained from the local travel unit, or the Communicable Disease Surveillance Centre (CDSC).

\section{Wound treatment}

The rabies virus is easily killed by sunlight, soap, and drying. Wound care is central to the prevention of rabies infection. In experimental animals, rabies transmission can be almost completely prevented by local wound treatment given within the first 3 hours after exposure. ${ }^{21}$ This does not mean that immunoprophylaxis can be ignored or avoided, but the risks and needs are greatly reduced by appropriate wound care. If faced with a wilderness injury away from appropriate medical care, adequate wound cleaning can greatly reduce the risks of infection.

Where possible, wound care should include infiltration with local anaesthetic. The wound should then be thoroughly scrubbed with water and either iodine solution, 40-70\% alcohol, or quaternary ammonium compounds (cetrimide $0.1 \% \mathrm{BPC}$ ), all of which have a proven lethal effect on the rabies virus.
During wound care, the edges of the wound must be scrubbed and any puncture wound must be cleaned thoroughly including the deepest parts. After scrubbing, the wound should be thoroughly rinsed with saline and covered with a simple dressing. Appropriate antibiotics should be used if indicated.

\section{Post-exposure prophylaxis}

The 1997 WHO survey indicated that in Europe alone 50742 individuals were given post-exposure prophylaxis following exposure to either domestic or wild animals. There were 13 European deaths from rabies during the same period, 10 of these occurring in the Russian Federation. Post-exposure prophylaxis involves a multifaceted approach combining wound care, passive treatment with immunoglobulin, and active vaccination.

The aim of post-exposure prophylaxis is to neutralise inoculated virus before it can enter the nervous system of the patient. Although time is of the essence, there is a definite window of up to 2 hours before detrimental effects ensue. During this time, advice can be obtained. Such advice is available from the Virus Reference Division of the Central Public Health Laboratory.

\section{Passive immunisation}

This is achieved using human rabies immunoglobulin (HRIG) which functions by neutralizing the rabies virus both locally and systemically within the first week prior to the body's own response to the vaccine. HRIG also appears to enhance the patient's $\mathrm{T}$ cell response to the vaccine. The initial dose of immunoglobulin is $20 \mathrm{IU} / \mathrm{kg}$ body weight, ${ }^{26}$ which should be infiltrated around the wound site as much as is anatomically feasible, the remainder being injected intramuscularly into an area as far as possible from the vaccination site. If HRIG is unavailable, WHO recommendations are to use equine immunoglobulin at a dose of $40 \mathrm{IU} / \mathrm{kg}$. Technically immunoglobulin should be given in all previously unvaccinated cases but it is especially important in those identified as at severe risk of infection (particularly head and neck wounds or multiple deep bites). Hypersensitivity reactions will occur in between 1 and $6 \%$ of individuals, and resuscitation equipment must be available at all times.

Unfortunately the HRIG is very expensive ( $£ 330$ for a $70 \mathrm{~kg}$ patient), ${ }^{27}$ and as such is not often available in the third world countries that carry the highest risk rabid animal injuries. Equine or ovine rabies immunoglobulin may be available, but they carry an allergic risk.

\section{Vaccination in the UK}

This usually involves the human diploid cell vaccine (Aventis) or an equivalent chick cell (Rabipur). Both are now licensed in the UK. Aventis is a freeze dried suspension of a Wistar rabies virus strain cultured in human diploid cells and inactivated by beta propiolactone. The protocol varies depending on whether a patient has had a previous full course of vaccine or not. Fully vaccinated patients should receive a booster vaccine on days 0 and 3 , into the deltoid muscle $^{28}$; this site has been shown to produce the best antibody titre available. Unvaccinated patients should receive doses on days $0,3,7,14$ and 28 . The full course need not be given in the $\mathrm{ED}$, but adequate arrangements must be made for follow up with the general practitioner.

Patients given the current vaccine reported approximately $30-74 \%$ levels of pain, swelling, erythema, and itching around the injection site. ${ }^{27}$ Systemic reactions such as headache, nausea, and abdominal pains were reported in $5-40 \%$ of recipients, most commonly in those patients receiving more frequent boosters. The overall anaphylaxis rates is $0.1 \%{ }^{29}$ 
In those patients currently taking immunosuppressive medications, these should be discontinued if at all possible to improve the immune response to the vaccine. Pregnancy is not a contraindication to giving either the vaccine or immunoglobulin, and the vaccine dosage is the same for children and adults. ${ }^{30}$

All known treatment failures that have occurred since 1980 have resulted from a deviation from the full recommended regimen. There are two reported cases of rabies where immunoglobulin and vaccine were injected into the gluteal area rather than the deltoid; it is assumed that subcutaneous fat interfered with the uptake, as occurs with the hepatitis B vaccine. $^{31}$

\section{Worldwide practice}

The "western" approach, although giving maximum protection, is very expensive. A dose of HRIG will cost approx $£ 320$ for the average male patient and vaccine is about $£ 17$ per adult dose. This puts such post-exposure treatment well beyond the cost of the majority developing world patients. A study of dog bite victims in Bangalore showed that among patients bitten and technically at risk of acquiring rabies, $86 \%$ received appropriate vaccination but none received the immunoglobulin. ${ }^{32}$ In Thailand between 1996 and 2001, fewer than $3 \%$ of those exposed received rabies immunoglobulin. ${ }^{33}$ The vaccine used is often Verorab, which is cheaper than other cell based vaccines but is equivalent to them.

Owing to the issues of expense, there are currently other regimens used throughout the world, which have been approved by the WHO in an attempt to provide the best possible care at low cost. An example currently used in Thailand uses eight injections of $0.1 \mathrm{ml}$ of vaccine at various sites on day 0 , four injections of $0.1 \mathrm{ml}$ on day 7 , and a single intradermal injection of $0.1 \mathrm{ml}$ on days 28 and 91. It uses approximately $40 \%$ of the vaccine required in the western regimen and appears to produce a very rapid antibody response with no reduction in effectiveness. ${ }^{33}$ Using smaller amounts of vaccine intradermally rather than intramuscularly, when there is no access to immunoglobulin, enables treatment of a greater proportion of the population while reducing the cost by approximately $60 \%{ }^{34}$ However, there have been reported accounts of treatment failure with this regimen. A 7 year old Thai girl was bitten by a suspected rabid dog, given the intradermal vaccine doses but unfortunately later developed and died from rabies encephalitis. Whether this could have been avoided with HRIG is unclear. ${ }^{35}$ Intradermal injections must be given correctly if they are to be used.

It is advisable that if any travellers returning to the UK have received treatment other than wound care overseas, accurate attempts be made to document the exact regimen they have been given. If necessary, advice can be sought from the health protection agency concerning local policies worldwide.

Current recommendations for rabies exposure management in developing countries include: ${ }^{36}$

- Publicising the urgency and efficacy of wound cleaning.

- Facilitating the replacement of nervous tissue vaccines by economical intradermal treatment with tissue cell vaccines.

- Using an intradermal regimen with a large dose of vaccine on the first day of treatment, especially when no immunoglobulin is available.

- Promoting pre-exposure prophylaxis, to eliminate the need for immunoglobulin and provide better rabies prophylaxis.
The lack of funding available in most developing countries has prompted a shift in thinking towards dog population control measures and canine vaccination, in an attempt to reduce the disease load to which patients are exposed. ${ }^{37} \mathrm{~A}$ programme for the mass vaccination of cats and dogs has taken place in Thailand, seeking to reduce the prevalence of the disease. ${ }^{38}$

\section{Who actually needs this treatment}

The central issues in the ED are which patients require postexposure treatment, and which regimen is the most appropriate. The following guidelines aim to give an idea of where to start but we recommend always discussing the case with the virologist on call in the local hospital for more specialist advice concerning the local protocol. We would recommend (a) consulting Virus Reference Division (WRD) or WHO literature for current information of rabies risk countries; $(b)$ determining the information in the rabies exposure form (Appendix A); and (c) contacting the on-call virologist, or if not available, the VRD of the Central Public Health Laboratory (CPHL) (Appendix B).

\section{CONCLUSION}

Rabies continues to kill large numbers of people throughout the world, although the disease is rarely seen in Britons and very rarely acquired within the UK. In the ED, the most likely contact with the disease will be in a worker or traveller who suspects that they have been exposed to the disease abroad. Important information must be obtained from the victim or a relation, regarding pre-exposure prophylaxis, nature of the exposure, and wound care undertaken. Details of any preexposure or post-exposure prophylaxis already given must also be recorded.

In most cases, the information obtained can be passed on to the local virology service who will advise appropriately. If this advice is unobtainable, the virus reference division of the CPHL can be contacted.

\section{ACKNOWLEDGEMENTS}

We thank Professors Mortimer and Greaves for their advice and critique.

\section{Authors' affiliations}

N McKay, Royal Gwent Hospital, Newport

L Wallis, Emergency Medicine, Cape Town, South Africa

Competing interest: there are no competing interests

\section{REFERENCES}

1 Chomel BB. The modern epidemiological aspects of rabies in the world. Comp Immunol Microbiol Inefect Dis 1993;16:11.

2 Rotivel Y, Goudal M, Wirth S, et al. (Rabies is a risk for travelling children). Arch Pediatr 1998:5:561-7.

3 Pounder D. Bat rabies. BMJ 2003;326:729.

4 Fooks AR, McElhinney LM, Pounder DJ, et al. Case report: isolation of a European bat lyssavirus type 2 a from a fatal human case of rabies encephalitis. J Med Virol 2003;71:281-9.

5 World Health Organisation Department of Communicable Disease, Surveillance and Response. World survey of rabies, no 33, for the year 1997. Geneva: WHO, 1999.

6 Sikes E. pathogenesis of rabies in wildlife. Comparative effect of varying doses of rabies virus inoculated into foxes and skunks. Am J Vet Res 1962;23:1041.

7 Eng T, Fishbein D. Epidemiologic factors, clinical findings and vaccination status of rabies in cats and dogs in the USA. National Study Group on Rabies. $J$ Am Vet Med Assoc 1990; 197:201.

8 Chhabra M, Ichhpujani RL. Animal bites: the current management guidelines. Indian J Pediatr 2003;70(suppl 1):S11-16.

9 Lurnio J, Hillborn M, Roine R, et al. Human rabies of bat origin in europe. Lancet 1986;1:378

10 Fishbein D, Robinson L. Current Concepts:rabies. New Engl J Med 1993;329:1632.

11 Houff SA, Burton RC, Wilson RW, et al. Human-to-human transmissiom of rabies virus by corneal transplant. N Engl J Med 1979;300:603-4.

12 Winkler WG, Fashinell TR, Leffingwell L, et al. Airborne rabies transmission in a laboratory worker. JAMA 1973;226:1219-21. 


\section{APPENDIX A FORM FOR POSSIBLE RABIES \\ EXPOSURE}

Date Time

Patient name:

Address:

Tel home:

Tel work:

GP and Tel:

Details of bite:

Country and Town:

Date of bite

Date of travel:

Nature of exposure: bite/lick/saliva/scratch/other

Location of exposure:

Was the skin broken? Yes/No

Did the wound bleed? Yes/No

Depth of bite: superficial/deep

Details of animal

Type of animal/species:

Wild/domestic (please delete)

Provoked/unprovoked (give details):

Is the animal's owner/home known? Yes/No

Efforts made to trace the animal? Yes/No

When was the animal last seen alive?

Animal's vaccination status, if known:

Patient's vaccination history

Did they have a 3-dose IM pre-exposure course? Yes/No

Details:

Was anti-rabies treatment given in this case? Yes/No

Was immunoglobulin given? Yes/No

Locally/systemically

Which vaccine was given?

Details (day/date etc.):

Other information:

Contact on call virologist for advice with above information.

If unavailable contact Virus Reference Division of the

Central Public Health Laboratory-02082004400.

Recommended treatment

Modified course for those with full pre- exposure

vaccination: days $0,3,7$

Standard course for unvaccinated: days $0,3,7,14,30$

Immunoglobulin $20 \mu \mathrm{g} / \mathrm{kg}$

Pt weight: Dose given:

Injection site:

Post-exposure course arranged? YES/NO

GP informed via letter? YES/NO

Name and signature of completing clinician

13 Dutta JK. Rabies transmission by oral and other non-bite routes. J Indian Med Assoc 1998;96:359.

14 Smith JS, et al. Unexplained rabies in three immigrants in the United States: a virological investigation. N Engl J Med 1991;324:205.

15 Jackson AC, Warrell MJ, Rupprecht CE, et al. Management of rabies in humans. Clin Infect Dis 2003;36:60-3.

16 Delpietro HA, Russo RG. Ecological and epidemiologic aspects of the attacks by vampire bats and paralytic rabies in Argentina and analysis of the proposals carried out for their control. Rev Sci Tech 1996;15:971-84.

17 Huff JL, Barry PA. B-virus (Cercopithecine herpesvirus 1) infection in humans and macaques: potential for zoonotic disease. Emerg Infect Dis 2003;9:246-50.

\section{APPENDIX B ADDRESSES AND WEBSITES}

\section{Contact details}

- Virus Reference Division of the Central Public Health Laboratory: 02082004400

- The Travel Unit, Communicable Disease Surveillance Centre: 02082006868.

\section{Websites}

- www.who-rabies-bulletin.org

- www.hpa.org.uk

- http://cphl.phls.org.uk

- http://www.hpa.org.uk/infections/topics_az/rabies/ HPA_Rabies_protocol_August_2003.pdf

- http://www.hpa.org.uk/infections/topics_az/ immunoglobulin/immunoglobulinHandbook.pdf

- http://www.doh.gov.uk/rabies/ memorandumonrabies/index.htm

18 Smith JS. New aspects of rabies with emphasis on epidemiology, diagnosis and prevention of the disease in the United States. Clin Microbiol Rev 1996;9:166-76.

19 Crepin, et al. Intravitam diagnosis of human rabies by PCR using saliva and cerebrospinal fluid. J Cli Microbiol 1998;3:1117-21.

20 Fooks AR, McElhinney LM, Brookes SM, et al. Rabies antibody testing and the UK Pet Travel Scheme. Vet Rec 2002;150:428-30.

21 Dean D, Baer G, Thowpson W. Studies on the local treatment of rabies infected wounds. Bull World Health Organ 1963;28:477.

22 Anderson L, Nicholson K, Tauxe R. Human rabies in the US 1960-79. Ann Intern Med 1984; 100:728-35

23 Strady C, Hung Nguyen V, Jaussaud R, et al. Pre-exposure rabies vaccination: strategies and cost-minimization study. Vaccine 2001;19:1416-24.

24 Dreesen DW, Hanlon CA. Current recommendations for the prophylaxis and treatment of rabies. Drugs 1998;56:801-9.

25 Centers for Disease Control. Human rabies prevention: United States, 1999: recommendations of the Advisory Committee on Immunization Practices (ACIP). MMWR Morb Mortal Wkly Rep 1999;48:1-28.

26 World Health Organization. Guidelines for post exposure treatment, 8th Report of the WHO Expert Commitee on Rabies. Geneva: WHO, 2000.

27 Bukata WR. Rabies-Essential facts for primary care physicians. Emerg Med Acute Care Essays 1999;23.

28 Baer G. Animal models in the pathogenesis and treatment of rabies. Rev Infect Dis 1988;10(suppl 4):S738

29 National Association of State Public Health Veterinarians, Inc. Compendium of animal rabies prevention and control, 2003. MMWR Recomm Rep 2003;52:1-6.

30 Sur DK, Wallis DH, O'Connell TX. Vaccinations in pregnancy. Am Fam Physician 2003;68:299-304.

31 Lumbiganon $\mathbf{P}$, Bunyahotra $\mathrm{V}$. Human rabies despite treatment with rabies immune globulin and human diploid cell rabies vaccine. JAMA 1988;259:25.

32 Sudarshan MK, Mahendra BJ, Narayan DH. A community survey of dog bites, anti-rabies treatment, rabies and dog population management in Bangalore city. J Commun Dis 2001;33:245-51

33 Kamoltham T, Singhsa J, Promsaranee U, et al. Elimination of human rabies in a canine endemic province in Thailand: five-year programme. Bull World Health Organ 2003;81:375-81.

34 Dutta JK. Human rabies in India: epidemiological features, management and current methods of prevention. Trop Doct 1999;29:196-201.

35 Sriaroon C, Daviratanasilpa S, Sansomranjai $P$, et al. Rabies in a Thai child treated with the eight-site post-exposure regimen without rabies immune globulin. Vaccine 2003;21:3525-6.

36 Warrell MJ. The challenge to provide affordable rabies post-exposure treatment. Vaccine 2003;21:706-9.

37 Wilde H, Briggs DJ, Meslin FX, et al. Rabies update for travel medicine advisors. Clin Infect Dis 2003;37:96-100.

38 Mitmoonpitak C, Tepsumethanon V, Wilde H. Rabies in Thailand. Epidemiol Infect 1998;120:165-9. 